\title{
Citation Diversity Statement in BMES Journals
}

\author{
(Published online 22 February 2021)
}

The Biomedical Engineering Society (BMES) is dedicated to fostering an inclusive environment and strives to represent all members of the biomedical engineering (BME) profession. To align with that goal, as editors of BMES journals, we have decided to include an optional citation diversity statement in our paper formats.

Gender and racial imbalances exist in a variety of academic fields, with the greatest disparities at the highest faculty positions., ${ }^{2,15,17}$ Several factors contribute to these imbalances, including caretaker responsibilities often falling to women, inadequate outreach, and too few role models for different genders, races, and ethnicities within a field of study. ${ }^{2,5,15}$ However, underlying biases also exist at both institutional and individual levels that impede progress in diversifying academia. ${ }^{8,13}$ These biases can affect employment opportunities, salaries, funding, and career advancement.

BME has a higher proportion of women than the engineering average for both bachelor's and doctoral degrees awarded and tenured or tenure track faculty. ${ }^{5}$ However, there is still room for a great deal of improvement, especially at the faculty level, which is only $23 \%$ female. ${ }^{14}$ In terms of racial and ethnic diversity, BME struggles to recruit diverse students and faculty, particularly those who are Black or African American, Hispanic, and American Indian or Hawaiian/Pacific Islander. ${ }^{5}$ Diversity in BME is not only important from an ethical standpoint to ensure that individuals are not discriminated against, but also because the entire field will benefit from the inclusion of people from different backgrounds. Diverse teams are better problem-solvers and can provide new insights that advance the field further. ${ }^{6,10}$ Also, having mentors and role models from historically underrepresented groups are crucial for the recruitment and success of new students.

One manifestation of bias in academia is the undercitation of publications authored by women and minoritized groups. Studies in a variety of fields have shown citation imbalances across gender, race, and ethnicity. $3,4,7,9,11,12$ Most of these studies evaluated gender imbalances and found that papers with female authors were cited less than those with male authors when controlling for several factors including year, publication venue, and paper focus. ${ }^{4,7,9,11,12}$ The citation patterns of male authors drove these observations, and in some fields imbalances have increased over time. ${ }^{9}$ Citation patterns based on race and ethnicity are more difficult to quantify based on author names. However, one study evaluated these patterns and found that papers with White first and last authors were cited more frequently in neuroscience journals. ${ }^{3}$ While many authors are likely unaware of these citation biases, intentional or unintentional bias introduced by a few authors can propagate, as more citations result in increased visibility. ${ }^{8}$

The inclusion of a citation diversity statement has been proposed to increase awareness and help correct citation imbalances. ${ }^{9}$ These statements should include the proportion of references cited by gender and/or race and ethnicity and appear directly before the reference list. Citation diversity statements serve two purposes. First, by analyzing the authorship of papers included in their reference list, authors may attempt to improve their citation balance. Second, the statements can draw readers' attention to the problem of citation imbalances and hopefully motivate them to re-evaluate their own work.

All BMES journals (Annals of Biomedical Engineering, Cellular and Molecular Bioengineering, Cardiovascular Engineering and Technology, and Biomedical Engineering Education) will now encourage authors to include a citation diversity statement within their manuscripts to work towards correcting citation imbalances in BME literature. The citation diversity statement is not required, but its inclusion will benefit authors, readers, and researchers from underrepresented demographics within BME. We also encourage publishers and ORCID to include the option to self-identify gender and race in the author and reviewer categories. As with all of our processes, we look forward to feedback for our readership.

The citation diversity statement should appear before the references in a manuscript. Its structure should include the following four parts: (1) the importance and motivation behind citation diversity, (2) the pro- 
portion of citations by gender and race/ethnicity for the first and last authors, (3) the method used to determine those proportions and its limitations, and (4) steps taken to by the authors to improve citation diversity in the article. ${ }^{19}$ The proportions of authors by gender should be divided into four categories based on first/last author combinations: woman/woman, man/woman, woman/man, and man/man. Race and ethnicity proportions should similarly be divided into four categories based on first/last author combinations: author of color/author of color, white author/author of color, author of color/white author, white author/ white author. Please see the example below based on the references in this paper. We recognize that one limitation of the proposed method is that it cannot account for intersex, non-binary, and transgender people, or Indigenous and mixed-race authors. One example of software used to determine author proportions for gender and race/ethnicity can be found at https://zenodo.org/record/4104748\#.X784zc1KiUm.

Given the current limitations of gender and race determinations, some authors may opt to not include a diversity statement. Alternatively an abbreviated statement can reasonably be substituted for the full statement as outlined above. Three representative examples, which should appear before the references in the manuscript with a subheading, are as follows:

\section{Option 1: No Statement}

Authors have the option to not include a citation diversity statement.

\section{Option 2: Abbreviated Statement}

Citation Diversity StatementRecent work in several fields of science has identified a bias in citation practices such that papers from women and other minority scholars are undercited relative to the number of papers in the field. ${ }^{4,7,9,11,12}$ We recognize this bias and have worked diligently to ensure that we are referencing appropriate papers with fair gender and racial author inclusion.

\section{Option 3: Full Statement}

Citation Diversity StatementRecent work in several fields of science has identified a bias in citation practices such that papers from women and other minority scholars are undercited relative to the number of papers in the field. ${ }^{4,7,9,11,12}$ Here, we sought to proactively consider choosing references that reflect the diversity of the field in thought, form of contribution, gender, race, ethnicity, and other factors. First, we obtained the predicted gender of the first and last author of each reference by using databases that store the probability of a first name being carried by a woman. ${ }^{9,18}$ By this measure (and excluding self-citations to the first and last authors of our current paper), our references contain 31\% woman(first)/woman(last), 31\% man/woman, 19\% woman $/ \mathrm{man}$, and $19 \% \mathrm{man} / \mathrm{man}$. This method is limited in that a) names, pronouns, and social media profiles used to construct the databases may not, in every case, be indicative of gender identity and b) it cannot account for intersex, non-binary, or transgender people. Second, we obtained predicted racial/ethnic category of the first and last author of each reference by databases that store the probability of a first and last name being carried by an author of color. ${ }^{1,16}$ By this measure (and excluding self-citations), our references contain $2 \%$ author of color (first)/author of color(last), 9\% white author/ author of color, $20 \%$ author of color/white author, and $69 \%$ white author/white author. This method is limited in that (a) names and Florida voter data used to make the predictions may not be indicative of racial/ethnic identity, and (b) it cannot account for Indigenous and mixed-race authors, or those who may face differential biases due to the ambiguous racialization or ethnicization of their names. We look forward to future work that could help us to better understand how to support equitable practices in science.

\section{REFERENCES}

${ }^{1}$ Ambekar, A., C. Ward, J. Mohammed, S. Male, and S. Skiena. Name-ethnicity classification from open sources. In: Proceedings of the 15th ACM SIGKDD International Conference on Knowledge Discovery and Data Mining, 2009, pp. 49-58.

${ }^{2}$ Beede, D. N., T. A. Julian, D. Langdon, G. McKittrick, B. Khan, and M. E. Doms. Women in STEM: a gender gap to innovation. Economics and Statistics Administration Issue Brief 2011. https://doi.org/10.2139/ssrn.1964782.

${ }^{3}$ Bertolero, M. A., J. D. Dworkin, S. U. David, C. L. Lloreda, P. Srivastava, J. Stiso, D. Zhou, K. Dzirasa, D. A. Fair, and A. N. Kaczkurkin. Racial and ethnic imbalance in neuroscience reference lists and intersections with gender. bioRxiv 2020. https://doi.org/10.1101/2020.10.12. 336230.

${ }^{4}$ Caplar, N., S. Tacchella, and S. Birrer. Quantitative evaluation of gender bias in astronomical publications from citation counts. Nat. Astron. 1:1-5, 2017.

${ }^{5}$ Chesler, N. C. A how-to guide for promoting diversity and inclusion in biomedical engineering. Ann. Biomed. Eng. 47:1167-1170, 2019.

${ }^{6}$ Chesler, N. C., G. Barabino, S. N. Bhatia, and R. Richards-Kortum. The pipeline still leaks and more than you 
think: a status report on gender diversity in biomedical engineering. Ann. Biomed. Eng. 38:1928-1935, 2010.

${ }^{7}$ Dion, M. L., J. L. Sumner, and S. M. Mitchell. Gendered citation patterns across political science and social science methodology fields. Polit. Anal. 26:312-327, 2018.

${ }^{8}$ Dworkin, J., P. Zurn, and D. S. Bassett. (In) citing action to realize an equitable future. Neuron 106:890-894, 2020.

${ }^{9}$ Dworkin J. D., K. A. Linn, E. G. Teich, P. Zurn, R. T. Shinohara, and D. S. Bassett. The extent and drivers of gender imbalance in neuroscience reference lists. arXiv preprint. arXiv:2001.01002, 2020.

${ }^{10}$ Hong, L., and S. E. Page. Groups of diverse problem solvers can outperform groups of high-ability problem solvers. Proc. Natl Acad. Sci. U.S.A. 101:16385-16389, 2004.

${ }^{11}$ Maliniak, D., R. Powers, and B. F. Walter. The gender citation gap in international relations. Int. Organ. 67:889922, 2013.

${ }^{12}$ Mitchell, S. M., S. Lange, and H. Brus. Gendered citation patterns in international relations journals. Int. Stud. Perspect. 14:485-492, 2013.

${ }^{13}$ Moss-Racusin, C. A., J. F. Dovidio, V. L. Brescoll, M. J. Graham, and J. Handelsman. Science faculty's subtle gender biases favor male students. Proc. Natl Acad. Sci. U.S.A. 109:16474-16479, 2012.

${ }^{14}$ Roy, J. Engineering by the numbers. In: American Society for Engineering Education 2019, pp. 1-40.

${ }^{15}$ Sidhu, R., P. Rajashekhar, V. L. Lavin, J. Parry, J. Attwood, A. Holdcroft, and D. S. Sanders. The gender imbalance in academic medicine: a study of female authorship in the United Kingdom. J. R. Soc. Med. 102:337-342, 2009

${ }^{16}$ Sood, G., and S. Laohaprapanon. Predicting race and ethnicity from the sequence of characters in a name. arXiv preprint. arXiv:1805.02109, 2018.

${ }^{17}$ Waisbren, S. E., H. Bowles, T. Hasan, K. H. Zou, S. J. Emans, C. Goldberg, S. Gould, D. Levine, E. Lieberman, and M. Loeken. Gender differences in research grant applications and funding outcomes for medical school faculty. J. Women's Health 17:207-214, 2008.

${ }^{18}$ Zhou, D., E. Cornblath, J. Stiso, E. Teich, J. Dworkin, A. Blevins, and D. Bassett. Gender Diversity Statement and Code Notebook, v1.0. https://doi.org/10.5281/zenodo. 3672110, 2020.

${ }^{19}$ Zurn, P., D. S. Bassett, and N. C. Rust. The citation diversity statement: a practice of transparency, a way of life. Trends Cogn. Sci. 24:669-672, 2020.

\section{Stefan M. Duma and Bethany Rowson Virginia Tech \\ Electronic mail: Duma@vt.edu}

Michael R. King

Vanderbilt University, Nashville, USA

IGOR Efimov

George Washington University, Washington, USA

\section{Ann SATERbak \\ Duke University, Durham, USA}

NAOMI C. ChesLer

University of California, Irvine, Irvine, USA

Publisher's Note Springer Nature remains neutral with regard to jurisdictional claims in published maps and institutional affiliations. 